\title{
A CINCUENTA AÑOS DE LA CREACIÓN DEL MINISTERIO DE CULTURA EN COSTA RICA. LA INVERSIÓN E INTERVENCIÓN ESTATAL EN EL ÁMBITO CULTURAL
}

\author{
Fifty years from the creation of the Ministry of Culture in Costa Rica. \\ State investment and state intervention in culture \\ Yanina Ruíz Siles \\ Universidad de Costa Rica, Costa Rica \\ yanina.ruiz@ucr.ac.cr
}

Recibido: 21-05-2020

Aprobado: 24-07-2020

\begin{abstract}
Yanina Ruíz Siles es licenciada en Historia por la Universidad de Costa Rica (UCR) y egresada de la Maestría en Historia Aplicada de la Universidad Nacional (UNA). Ha laborado como docente en la Universidad de Costa Rica (UCR), la Universidad Nacional Estatal a Distancia (UNED) y la Universidad Técnica Nacional (UTN).
\end{abstract}

\section{RESUMEN}

El desarrollo de la institucionalidad cultural costarricense data del siglo XIX, pero a partir de la creación del MCJD se dio una centralización en la inversión e intervención estatal en este ámbito. Permitiendo así, que el sector cultural se expandiera durante la década de 1970 y 1980 . Sin embargo, la adopción del modelo neoliberal tuvo un impacto negativo en este crecimiento, el cual se ve plasmado entre los años 1990 y 2020. El presente trabajo tiene como objetivo analizar estos procesos en Costa Rica, para ello se revisaron las memorias institucionales y las leyes de presupuesto del Ministerio de 1971 al 2020. En el artículo se observan las continuidades y transformaciones de esta entidad.

Palabras clave: historia cultural; Ministerio de Cultura; institucionalidad cultural; políticas culturales; inversión estatal; intervención estatal; Costa Rica

\begin{abstract}
The development of the Costa Rican cultural institutionality dates from the 19th century, but from the creation of the MCJD there was a centralization in state investment and intervention in this area. Thus, allowing the cultural sector to expand during the 1970s and 1980s. However, the adoption of the neoliberal model had a negative impact on this growth, which is reflected between the years 1990 and 2020 . The present work aims to analyze these processes in Costa Rica, the institutional reports and budget laws of the Ministry from 1971 to 2020 were reviewed. The article shows the continuities and transformations of this entity.
\end{abstract}

Keywords: cultural history; Ministry of Culture; cultural institutionality; cultural policies; state investment; state intervention; Costa Rica 


\section{Introducción}

La cultura son todos aquellos conocimientos y prácticas, explícitos e implícitos que regulan el comportamiento de una sociedad. Son los hábitos, normas, códigos, creencias y rituales que caracterizan a una comunidad. La acepción del término es compleja, y por ello su institucionalidad debe contemplar una serie de aristas.

En Costa Rica, los procesos de institucionalidad cultural han estado marcados por fluctuaciones significativas, y han atravesado diferentes etapas. La intervención estatal en materia cultural inició después de la independencia, como una respuesta ante la necesidad de generar una integración entre los costarricenses. Estos procesos se siguieron dando a lo largo de la historia, y los picos de inversión están claramente acentuados por crisis políticas o económicas. De esta forma, el sector cultural costarricense ha sido utilizado como una herramienta gubernamental para legitimar al gobierno. Las instituciones surgen como un recurso que facilita el adoctrinamiento y limita los espacios de ocio.

A partir de 1970, con la instauración del Ministerio de Cultura, Juventud y Deportes (MCJD), se agilizó el proceso de institucionalización, al mismo tiempo que se creaba una legislación cultural, que a la postre permitió la creación, regulación y manutención de estas entidades.

Este trabajo busca analizar el desarrollo cultural del país, por medio de las políticas y financiamiento estatal. Con este objetivo, se dividirá el análisis en cuatro apartados: en un primer apartado se analizarán las primeras propuestas que se plantearon para crear un Ministerio. Después, se estudiará la creación del MCJD y la edad dorada en el sector cultural, para terminar con la retracción del aparato burocrático en la década de los noventas. Seguidamente, se investigará el impacto que ha tenido la reducción de presupuesto y la separación de Deportes del Ministerio. Finamente, se examinarán los cambios en la intervención gubernamental durante los cincuenta años del Ministerio.

Las fuentes analizadas para este trabajo comprenden Memorias, Informes de Labores y Leyes asociadas con el ámbito cultural. Estas referencias brindan información clave a fin de desarrollar la investigación, ya que se puede extraer datos relativos a la inversión e intervención gubernamental. Con esto se determinarán las estrategias estatales durante el período de estudio. 
La metodología es mixta. El análisis cualitativo consiste en una triangulación entre las fuentes primarias y secundarias, que permitirán visualizar el discurso oficial. Para la parte cuantitativa, se elaboró una base de datos, que identifica la inversión estatal, y los sectores donde se da una mayor intervención.

La presente investigación, busca generar un aporte para la historia cultural, realizando un énfasis en lo acontecido en materia cultural durante el siglo XXI. Ya que la mayoría de los estudios historiográficos sobre este tema se enfocan en los siglos anteriores.

\section{Institucionalización de la cultura}

La década de 1940, fue una época convulsiva en Costa Rica. En este período, se dieron una serie de hechos que desencadenaron la formación de nuevas estructuras sociopolíticas. Estos eventos están asociados con la Segunda Guerra Mundial, y la Guerra Civil del 48. Consecuencia de estos acontecimientos se fundó la Segunda República. Para se estableció una Asamblea Constituyente, cuyo propósito era escribir una nueva constitución política.

En la Asamblea Constituyente, fue donde se mencionó por primera vez la necesidad de instaurar un órgano rector de la cultura. Esta idea surgió en medio de la discusión del capítulo denominado "La cultura" posteriormente llamado "La educación y la cultura". Asimismo, en medio de la disputa, como se observa en el tercer tomo de actas, y en relación con este debate, el diputado Mario Alberto Jiménez Quesada, señala que "La Educación es función esencial del Estado, el cual está en la obligación de crear las instituciones y servicios suficientes para atender a las necesidades educacionales y culturales del país." Y que "Entre los fines culturales de la República están el de conservar, desarrollar y nacionalizar la riqueza histórica y artística, y el de apoyar la iniciativa privada para el progreso científico y artístico del país." (pág. 5-6).

Considerando lo expuesto por el diputado Jiménez Quesada, es fácil comprender porque se tuvo que modificar el nombre del capítulo, pues, aunque indudablemente la educación es parte de la cultura, y es una herramienta para transmitirla, el título la superaba. De esta manera, se propuso la creación de una entidad independiente que vele únicamente por la cultura. Dicha propuesta fue apoyada por diferentes diputados, donde destacan Isaac Felipe Azofeifa Bolaños, Daniel Oduber Quirós y Carlos Monge Alfaro. 
El tema se vuelve controversial. No obstante, debido al contexto y los intereses gubernamentales la propuesta no prolifera. Pero, la adopción del modelo benefactor, y por consiguiente el crecimiento del aparato estatal cimenta las bases para la inversión cultural acontecida durante la segunda mitad del siglo $X X$.

A partir de 1950, Costa Rica entró en una fase de modernización e incremento económico. Consecuencia de este fenómeno se dio un aumento en flujo migratorio nacional, en ambas vías, impulsados por la colonización agrícola y el empleo asalariado. El crecimiento acelerado del área metropolitana tuvo repercusiones en el paisaje urbano, la disminución de zonas verdes y el deterioro de las identidades locales (Molina, 2002).

La urbanización trajo consigo una serie de cambios en la vida cotidiana de los vecinos del Valle Central, que se vio impactado por la pérdida de los espacios públicos, los avances en las telecomunicaciones y los medios de transporte, que favorecieron el cambio cultural. Paulatinamente, la cultura de masas estadounidense (American Way of Life) fue ganando espacio y suplantando algunos hábitos nacionales.

La amenaza que implicaba para la identidad nacional estas nuevas costumbres llevó al Estado a replantearse la idea de invertir cultura. En el decenio de 1960, se creó la Dirección General de Artes y Letras, y subsecuentemente se nombró a representantes en diversas instituciones culturales enfocadas la divulgación, protección y estimulación del arte. Con el aumento de la brecha social, emergió el descontento, las huelgas y las manifestaciones contra el gobierno (Cuevas, 1995).

En respuesta a esta convulsiva realidad en marzo de 1970 se debate nuevamente la propuesta de instaurar un Ministerio de Cultura. Cuyo propósito era crear una "sociedad culta". 


\section{Los "años dorados" de la cultura}

El 3 de diciembre de 1970, la Asamblea Legislativa aprobó la creación del MCJD, el cual inició sus funciones en enero de 1971, bajo el liderazgo de Alberto Cañas Escalante. El 5 de julio de 1971, mediante la Ley No.4788, se establecieron las funciones y objetivos de la entidad. Asimismo, se determinó cuales instituciones estarían adscritas al ente.

Los objetivos del Ministerio giraban en torno a estos cuatro ejes:

- Investigación, estaría a cargo de investigar todos los aspectos culturales e históricos de la sociedad costarricense para su rescate,

- Divulgación, daría a conocer toda la información obtenida mediante la investigación y difundiría el arte y la cultura en cada rincón del país,

- Descentralización, promovería la cultura a lo largo y ancho de todo el país, y finalmente

- Promoción, velaría por estimular y apoyar a los artistas costarricenses.

Tras su fundación, el Ministerio absorbe, bajo la Dirección General de Cultura a las siguientes instancias: el Departamento de Radio, el Departamento de Publicaciones, Departamento de Investigación del Folclore, el Departamento de Música, el Departamento de Defensa del Patrimonio Artístico y Cultural, Sistema Nacional de Bibliotecas, el Teatro Nacional, la Compañía Nacional de Teatro, la Dirección General de Artes y Letras, Editorial Costa Rica, la Orquesta Sinfónica y el Museo Nacional, la Casa del Artista, además de varios órganos desconcentrados.

Durante esta etapa, según señala el ministro Cañas Escalante, en la Memoria de 1972-1973:

He de confesar que en este ramo [cultura] es donde nuestra acción se hace más fácil. Era muy poco, poquísimo, lo que existía o se hacía en ese amplio campo de la actividad humana, y al mismo tiempo existe en todas las capas sociales del país, un anhelo de contacto con esas actividades. (pág. 5).

Debido a esta situación, el MCJD se da a la tarea de crear diversas instituciones durante la década de 1970, que tenían como objetivo abordar temas y cubrir a la población que los programas actuales no albergaban. En el siguiente cuadro se observan las entidades instauradas en este decenio. 
Cuadro 1. Entes creados por el MCJD en la década de 1970.

\begin{tabular}{|c|l|c|}
\hline Fundación & \multicolumn{1}{|c|}{ Ente } & Tipo \\
\hline 1973 & Departamento de Cine & Institución \\
\hline 1974 & Museo Histórico Cultural Juan Santamaría & Institución \\
\hline 1974 & Coro de la Sinfónica Nacional & Programa \\
\hline 1976 & Orquesta Sinfónica Juvenil & Programa \\
\hline 1977 & Museo de Arte Costarricense & Institución \\
\hline 1978 & Taller Nacional de Teatro & Proyecto \\
\hline 1979 & Compañía Nacional de Danza & Proyecto \\
\hline
\end{tabular}

Fuente: Elaboración propia, a partir Memorias del MCJD (1971-1979)

*Únicamente se contabilizaron las instituciones de cultura

Dentro de estas instituciones cabe destacar la creación del Museo Histórico Cultural Juan Santamaría ubicado en Alajuela, que es la primera entidad cultural creada por el Estado fuera de la capital.

La permanencia del Partido Liberación Nacional (1970-1978) por dos períodos en el poder, también contribuyó a que la inversión en cultura se mantuviera constante. Lo que le permitió al MCJD crear departamentos, direcciones y otras instituciones que facilitaron el cumplimiento de estos ejes. A finales de la década, se creó el Sistema Nacional de Radio y Televisión (SINART) y se trasladó al Ministerio temporalmente la Dirección Nacional de Desarrollo de la Comunidad (mejor conocido como DINADECO). El objetivo de esto era descentralizar la cultura y llegar a los rincones más lejanos del país (Cuevas, 1995).

El primer lustro de la década de 1980 estuvo marcado por la promulgación de leyes y decretos que favorecieron el desarrollo cultural, entre estos destacan la creación impuestos para el mantenimiento de las instituciones de cultura públicas, y la inversión estatal en arte. Consecuencia de la crisis económica y las reformas liberales, gradualmente el apoyo fue disminuyendo, y se empezaron a derogar leyes y decretos culturales. Debido a esto, como se observa en el siguiente cuadro, en este decenio se crearon pocos programas, si se compara con el anterior. No obstante, estos estuvieron más enfocados en la difusión cultural fuera del área metropolitana. 
Cuadro 2. Entes creados por el MCJD en la década de 1980.

\begin{tabular}{|c|l|c|}
\hline Fundación & \multicolumn{1}{|c|}{ Ente } & Tipo \\
\hline 1980 & Comités de Cultura & Programa \\
\hline 1980 & Colegio de Costa Rica & Programa \\
\hline 1980 & Compañía Lírica Nacional & Programa \\
\hline 1981 & Dirección General de Museos & Programa \\
\hline
\end{tabular}

Fuente: Elaboración propia, a partir Memorias del MCJD (1980-1989)

*Únicamente se contabilizaron las instituciones de cultura

Según se observa en el cuadro, en esta década no la inversión estatal no estuvo dirigida a la fundación de instituciones, sino que se enfocó en la creación de programas que apoyaran los emprendimientos culturales, un ejemplo de esto es la Dirección General de Museos. Asimismo, en este período también se crean los Comités de Cultura, cuyo objetivo era crear organizaciones cantonales que preservaran y fomentaran la cultura de las comunidades.

Recapitulando, durante los primeros veinte años la labor del MCJD estuvo enfocada en dos líneas, la conservación del patrimonio histórico cultural, y el desarrollo artístico. Es debido a esto que la mayoría de las instituciones y programas que se crean en esta etapa están relacionados con las bellas artes, el otro elemento que sobresale es la creación de museos y entidades para este fin.

\section{Reducción del aparato estatal}

En la década de 1990, el Estado implemento una serie de reformas, que permitieron la derogación y privatización de instituciones gubernamentales, con ello logran reducir el gasto público y retraer el aparato estatal. En materia de cultura, esto se ve reflejado en la reducción del financiamiento en cultura. No obstante, en este decenio se da un aumento en la creación de entidades mixtas, como museos y teatros (Zavaleta, 2013).

Durante este período los fondos de esta cartera pasaron de 1,2\% del PIB a 0,6\%, es decir, se redujeron en un $50 \%$ en un lapso de diez años. Debido a ello, y como se aprecia en el siguiente cuadro, en esta década únicamente se crearon tres museos, el resto de las instituciones se mantuvieron, exceptuando algún cambio de nombre realizado para que el título se apegará más a las actividades efectuadas por la entidad. 
Cuadro 3. Entes creados por el MCJD en la década de 1990.

\begin{tabular}{|c|l|c|}
\hline Fundación & \multicolumn{1}{|c|}{ Ente } & Tipo \\
\hline 1991 & Museo Dr. Rafael Ángel Calderón Guardia & Institución \\
\hline 1994 & Museo de Arte y Diseño Contemporáneo & Institución \\
\hline 1997 & Centro Cultural e Histórico José Figueres Ferrer & Institución \\
\hline
\end{tabular}

Fuente: Elaboración propia, a partir Memorias del MCJD (1990-1999)

*Únicamente se contabilizaron las instituciones de cultura

La retracción del Estado les abrió un portillo a las empresas privadas y extranjeras, que empezaron a invertir en cultura. Paralelamente, se impulsó el emprendimiento de los artistas, de manera que sus actividades no fuesen financiadas por el Estado. Asimismo, en 1998 mediante la Ley No.7800, se crea el Instituto Costarricense del Deporte y la Recreación (ICODER), lo que marca una separación de estas actividades del Ministerio de Cultura.

En la década de 2000, el presupuesto del MCJ se mantuvo en aproximadamente un $0,5 \%$ del PIB, lo que dificulto la creación de nuevas instituciones, en este período, como se observa en el cuadro 3, el Ministerio únicamente creo tres entidades, una de ellas es el Museo de Formas, Espacios y Sonidos, que cerró en el 2008, y la otra es el Programa de Museos Regionales y Comunitarios, que vino a suplir el vacío generado tras la clausura de la Dirección General de Museos.

Cuadro 4. Entes creados por el MCJD en la década de 2000.

\begin{tabular}{|c|l|c|}
\hline Fundación & \multicolumn{1}{|c|}{ Ente } & Tipo \\
\hline 2002 & Museo de Formas, Espacios y Sonidos & Institución \\
\hline 2002 & Programa de Museos Regionales y Comunitarios & Programa \\
\hline 2007 & Parque La Libertad & Proyecto \\
\hline
\end{tabular}

Fuente: Elaboración propia, a partir Memorias del MCJD (2000-2009)

*Únicamente se contabilizaron las instituciones de cultura 
En la década de 2010, los fondos volvieron a tener un descenso significativo, pasando de un $0,60 \%$ del PIB a un $0,10 \%$, siendo el presupuesto más bajo en toda la historia del MCJ. Debido a esta reducción en este período únicamente se crearon dos instituciones.

Cuadro 5. Entes creados por el MCJD en la década de 2010.

\begin{tabular}{|c|l|c|}
\hline Fundación & \multicolumn{1}{|c|}{ Ente } & Tipo \\
\hline 2010 & Sistema Nacional de Educación Musical & $\begin{array}{c}\text { Órgano } \\
\text { desconcentrado }\end{array}$ \\
\hline 2013 & Centro de Producción Artística y Cultural & Institución \\
\hline
\end{tabular}

Fuente: Elaboración propia, a partir Memorias del MCJD (2010-2019)

*Únicamente se contabilizaron las instituciones de cultura

No obstante, las instituciones creadas en este decenio no suplen ningún vacío, pues el Ministerio ya contaba con entidades enfocadas en estas temáticas, lo que podría considerarse una duplicidad de labores, y tampoco cubren a un sector nuevo de la población.

\section{Balance del desempeño del Ministerio de Cultura}

En la sección anterior se revisó la inversión estatal en cultura, a partir de esto se pudo percibir que desde la creación del MCJD el presupuesto de esta cartera se ha ido reduciendo considerable. El discurso del Estado es que los ingresos del MCJD ha tenido un aumento en lo que refiere números absolutas. Sin embargo, la realidad es que ha tenido una disminución en el porcentaje de ingresos que recibe del Producto Interno Bruto (PIB),

Como se observa en el gráfico 1, los fondos del Ministerio siempre han oscilado el $1 \%$ del PIB. Sin embargo, desde 1990 se percibe una reducción considerable, la cual se ha mantenido constante hasta llegar en el 2020 aún 0,1\%. 
Gráfico 1. Presupuesto del Ministerio de Cultura (1971-2020).

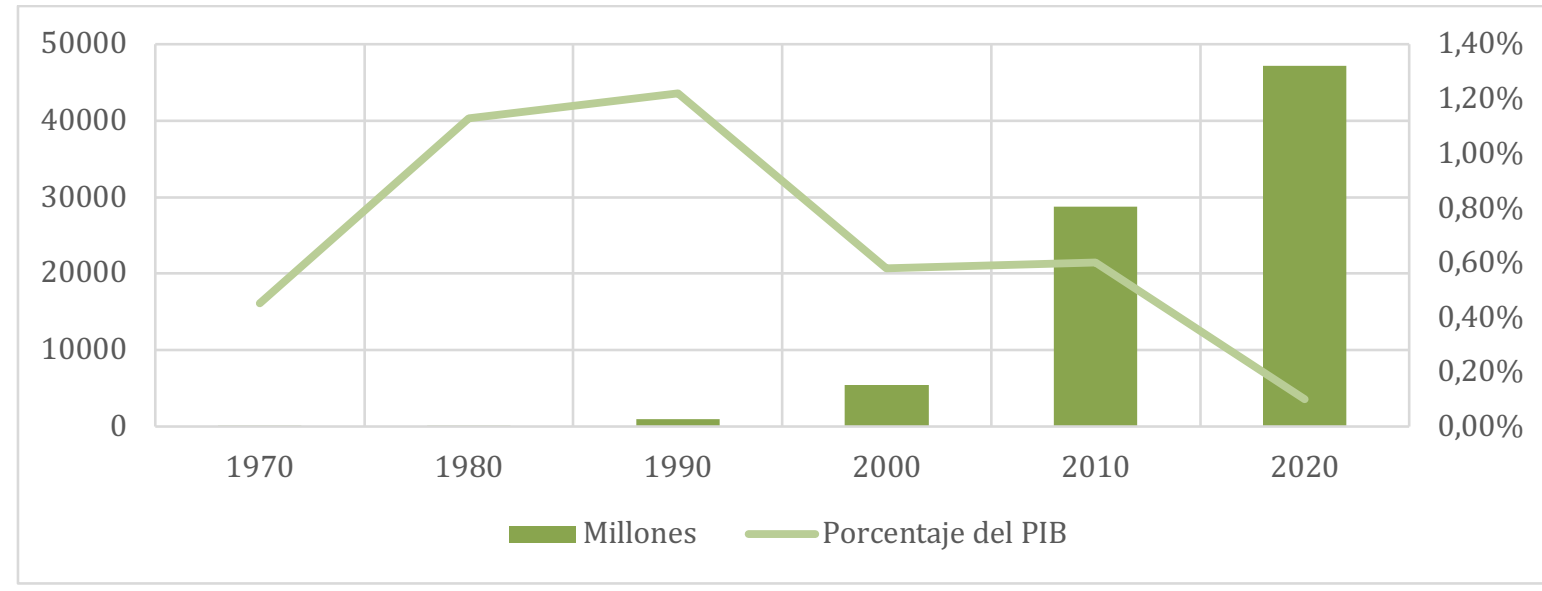

Fuente: Elaboración propia, a partir de Leyes de presupuestos ordinarios y extraordinarios MCJD (19712020)

*Hasta 1998 incluye Deportes.

La reducción en la inversión e intervención estatal en este y otros ámbitos es el resultado de la adopción del modelo neoliberal. Este proceso inició en la década de 1980, y se fue dando de forma gradual, por lo cual su impacto no se ve en algunos sectores hasta mediados del decenio de 1990. (Vargas, 2016) Las repercusiones en el sector cultura, fue uno de los primeros en percibirse, ya que al no tratarse de un aspecto "esencial" por lo cual se considera que es fácil de prescindir.

La disminución en los ingresos se ve reflejada en los programas e instituciones, pues como se aprecia en el siguiente gráfico desde la creación del MCJD se ha dado una reducción en la instauración de entes culturales. 
Gráfico 2. Creación estatal de entes culturales, por década (1971-2020).

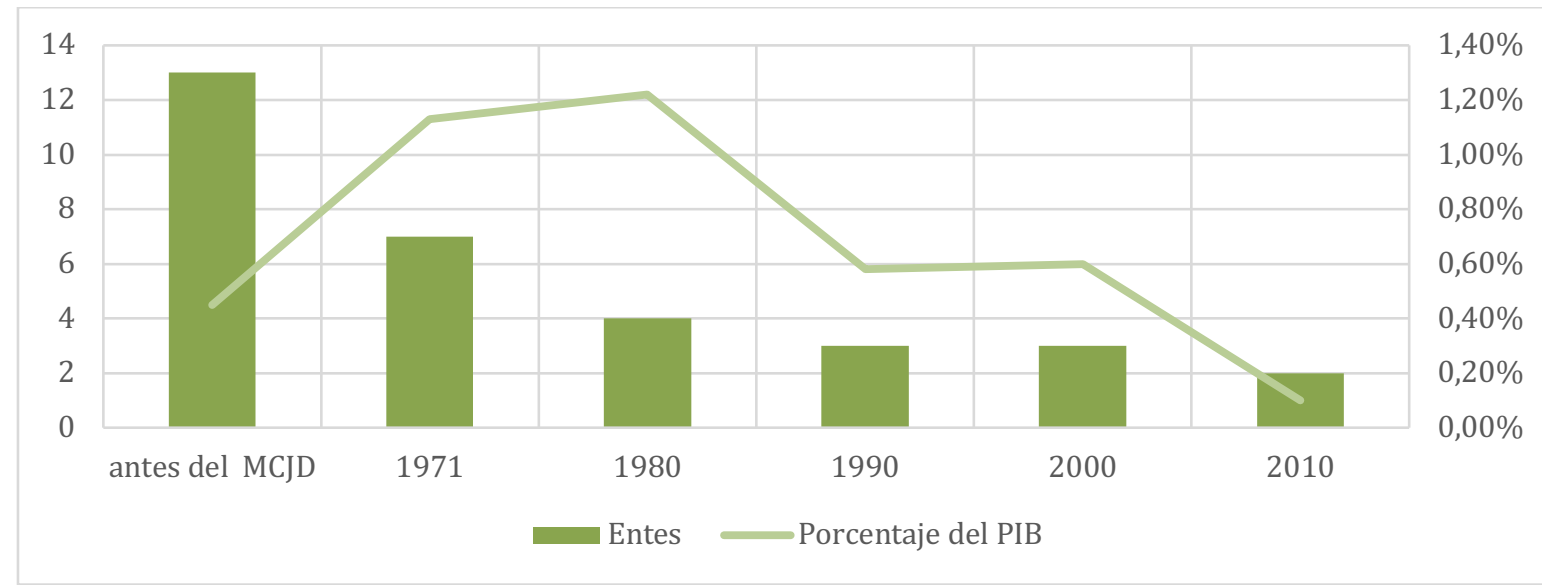

Fuente: Elaboración propia, a partir de Leyes de presupuestos ordinarios y extraordinarios MCJD y las Memorias del MCJD (1971-2020)

*Hasta 1998 incluye Deportes.

En el gráfico se aprecia que la mayoría de las instituciones estatales de cultura en el país fueron creadas antes de la creación del MCJD. Asimismo, la década de los setenta es donde se instauran más entes debido al vacío que existía de estas. Sin embargo, se observa cómo se da una disminución en esta intervención según se reduce el presupuesto.

\section{Conclusiones}

La creación del MCJD trajo al país cambios en esta materia, permitió la institucionalización y financiamiento de diferentes espacios que anteriormente no contaban con apoyo estatal, asimismo contribuyo significativamente en el rescate del patrimonio cultural. El Ministerio también promovió la descentralización, lo que impulsó la democratización de la cultura y ha facilitado la conservación del del folclore, la cultura popular y la historia local.

Como se observó a lo largo del trabajo, el desempeño de esta cartera depende principalmente del presupuesto, pues la mayoría de su oferta está conformada por proyectos y órganos desconcentrados, que no cuentan con muchos funcionarios. Esto quiere decir que, para tener un mayor alcance, requiere trasladarse a otras zonas del país donde no hay instituciones establecidas. Sin 
embargo, la reducción sistemática en el presupuesto de Cultura ha implicado que el MCJ tuviera que derogar o reducir el financiamiento a sus programas y entidades, lo que ha conlleva a un detrimento de la labor de este Ministerio, ya que esto implica que las personas deban de movilizarse a la capital para poder disfrutar de sus actividades.

Los fondos que el Ministerio asigna a sus instituciones no son suficientes para suplir con sus necesidades, esto ha llevado a un número significativo a recurrir a otro tipo de actividades, como el voluntariado, donaciones y la venta de servicios. Este último desvirtúa los principios con lo que el MCJ fue abierto, pues muchas de sus actividades no son gratuitas, lo que le imposibilita que algunos sectores de la población puedan disfrutar de estas puestas en escena.

\section{BIBLIOGRAFÍA}

\section{Fuentes primarias}

Asamblea Legislativa. (2008). Actas de la Asamblea Constituyente 1949. Tomo III-III. San José: EUNED.

Ministerio de Cultura, Juventud y Deportes. (1971-1998) Memorias institucionales.

Ministerio de Cultura y Juventud. (1999-2019). Memorias institucionales.

\section{Libros y artículos}

Cuevas, R. (1995). El punto sobre la i: Políticas culturales del estado costarricense, 1948-1990. San José: MCJD.

Molina, I. (2002). Costarricense por dicha: identidad nacional y cambio cultural en Costa Rica durante los siglos XIX y XX. San José: EUCR.

Vargas, L. P. (2016). El Proyecto Histórico Neoliberal en Costa Rica (1984-2015): Devenir histórico y crisis. Revista Rupturas 6(1), 147-162.

Zavaleta, E. (2013). La construcción del mercado del arte en Costa Rica: políticas culturales, acciones estatales, y colecciones públicas (1950-2005). San José: EUCR. 